\title{
THE RED SUPERGIANT PROGENITOR OF SUPERNOVA 2012aw (PTF12bvh) IN MESSIER 95
}

\author{
Schuyler D. Van Dyk ${ }^{1}$, S. Bradley Cenko ${ }^{2}$, Dovi Poznanski ${ }^{3}$, Iair Arcavi ${ }^{4}$, Avishay Gal-Yam ${ }^{4}$, Alexei V. Filippenko $^{2}$, \\ Kathryn Silverio ${ }^{2}$, Alan Stockton ${ }^{5}$, Jean-Charles Cuillandre ${ }^{6}$, Geoffrey W. Marcy ${ }^{2}$, \\ ANDREW W. HOWARD ${ }^{2}$, AND HOWARD ISAACSON ${ }^{2}$ \\ ${ }^{1}$ Spitzer Science Center/Caltech, Mailcode 220-6, Pasadena, CA 91125, USA; vandyk@ipac.caltech.edu \\ ${ }^{2}$ Department of Astronomy, University of California, Berkeley, CA 94720-3411, USA; cenko@ berkeley.edu, afilippenko@berkeley.edu, \\ silverio@astro.berkeley.edu, gmarcy@astro.berkeley.edu, howard@ astro.berkeley.edu, isaacson@astro.berkeley.edu \\ ${ }^{3}$ Faculty of Exact Sciences, Tel-Aviv University, 69978 Tel-Aviv-Yafo, Israel; dovi@wise.tau.ac.il \\ ${ }^{4}$ Department of Particle Physics and Astrophysics, Faculty of Physics, The Weizmann Institute of Science, \\ Rehovot 76100, Israel; iair.arcavi@weizmann.ac.il, avishay.gal-yam@weizmann.ac.il \\ ${ }^{5}$ Institute for Astronomy, University of Hawaii, 2680 Woodlawn Drive, Honolulu, HI 96822, USA; stockton@ @ifa.hawaii.edu \\ ${ }^{6}$ Canada-France-Hawaii Telescope Corporation, 65-1238 Mamalahoa Hwy, Kamuela, HI 96743, USA; jcc@ cfht.hawaii.edu \\ Received 2012 May 27; accepted 2012 July 11; published 2012 August 22
}

\begin{abstract}
We report on the direct detection and characterization of the probable red supergiant (RSG) progenitor of the intermediate-luminosity Type II-Plateau (II-P) supernova (SN) 2012aw in the nearby (10.0 Mpc) spiral galaxy Messier 95 (M95; NGC 3351). We have identified the star in both Hubble Space Telescope images of the host galaxy, obtained 17-18 yr prior to the explosion, and near-infrared ground-based images, obtained 6-12 yr prior to the SN. The luminous supergiant showed evidence for substantial circumstellar dust, manifested as excess line-of-sight extinction. The effective total-to-selective ratio of extinction to the star was $R_{V}^{\prime} \approx 4.35$, which is significantly different from that of diffuse interstellar dust (i.e., $R_{V}=3.1$ ), and the total extinction to the star was therefore, on average, $A_{V} \approx 3.1 \mathrm{mag}$. We find that the observed spectral energy distribution for the progenitor star is consistent with an effective temperature of $3600 \mathrm{~K}$ (spectral type M3), and that the star therefore had a bolometric magnitude of -8.29 . Through comparison with recent theoretical massive-star evolutionary tracks we can infer that the RSG progenitor had an initial mass $15 \lesssim M_{\text {ini }}\left(M_{\odot}\right)<20$. Interpolating by eye between the available tracks, we surmise that the star had initial mass $\sim 17-18 M_{\odot}$. The circumstellar dust around the progenitor must have been destroyed in the explosion, as the visual extinction to the $\mathrm{SN}$ is found to be low $\left(A_{V}=0.24\right.$ mag with $\left.R_{V}=3.1\right)$.
\end{abstract}

Key words: galaxies: individual (Messier 95, NGC 3351) - stars: evolution - stars: fundamental parameters stars: late-type - supernovae: general - supernovae: individual (SN 2012aw)

\section{INTRODUCTION}

Supernovae (SNe) are among the most powerful explosions in the universe. In addition to Type Ia SNe, which arise from the thermonuclear runaway explosion of a mass-accreting white dwarf star, there are $\mathrm{SNe}$ that result from the collapse of the core at the endpoint of a massive (with initial mass $M_{\text {ini }} \gtrsim 8 M_{\odot}$; e.g., Woosley \& Weaver 1986) star's evolution. If the star explodes with most of its extended hydrogen envelope still relatively intact, the event will be observed as a Type II-Plateau SN (SN II-P; Barbon et al. 1979). We would expect such a progenitor star to be in the red supergiant (RSG) phase at the time of core collapse.

We have been extremely fortunate in recent years to detect and characterize the probable RSG progenitors of SNe II-P in nearby galaxies. (We note that the most famous progenitor identification, of the star Sk $-69^{\circ} 202$ that exploded as SN 1987A in the Large Magellanic Cloud, was actually a blue supergiant, not an RSG; e.g., Arnett et al. 1989). One of the best examples is the identification in high-quality, ground-based imaging data of the RSG progenitor of the SN II-P 2008bk in NGC 7793 (Mattila et al. 2008; Van Dyk et al. 2012). Other SN II-P RSG progenitors have also been directly identified in archival Hubble Space Telescope (HST) images of nearby host galaxies, including SN 2003gd in M74 (Van Dyk et al. 2003; Smartt et al. 2004), SN 2004A in NGC 6207 (Hendry et al. 2006), SN 2005cs in M51 (Maund et al. 2005; Li et al. 2006), and SN 2009md in NGC 3389 (Fraser et al. 2011). All five of these SNe II-P are of low luminosity, with bolometric luminosi- ties $L_{\text {bol }} \lesssim 10^{41.5} \mathrm{erg} \mathrm{s}^{-1}$ at maximum, and with lower ejecta velocities during the plateau and lower luminosity on the lightcurve tail as a result of a smaller ${ }^{56} \mathrm{Ni}$ yield in the explosion (e.g., Zampieri et al. 2003; Pastorello et al. 2004). This is relative to intermediate-luminosity SNe II-P, such as SN 1999em in NGC 1637 (e.g., Hamuy et al. 2001; Leonard et al. 2002; Elmhamdi et al. 2003), with $L_{\text {bol }} \approx 10^{41.5}-10^{42} \mathrm{erg} \mathrm{s}^{-1}$ at maximum.

Three other SNe II-P also have direct probable progenitor identifications: SN 1999ev in NGC 4274 (Maund \& Smartt 2005), SN 2004et in NGC 6946 (Li et al. 2005; Crockett et al. 2011), and SN 2008cn in NGC 4608 (Elias-Rosa et al. 2009). SN 1999ev has no published photometry or spectroscopy, so its nature has not been well determined. SN 2008cn appears to have been similar to high-luminosity SNe II-P (with $L_{\text {bol }} \approx$ $10^{42.5} \mathrm{erg} \mathrm{s}^{-1}$ at maximum), such as SN 1992H (Clocchiatti et al. 1996) and SN 2007od (Inserra et al. 2011), which also exhibit a less pronounced plateau and more linear post-maximum decline. Furthermore, Elias-Rosa et al. (2009) detected a progenitor for SN 2008cn that was more yellow than red. Maguire et al. (2010) showed that SN 2004et bears similarities in both its photospheric expansion velocity and the overall shape of its bolometric light curve to those of SN 1999em (although SN 2004et may have been a factor of two more luminous than SN 1999em), implying that SN 2004et may also have been of intermediate luminosity. However, the nature and initial mass of the identified progenitor star have been debated (Li et al. 2005; Crockett et al. 2011; S. D. Van Dyk \& T. H. Jarrett 2012, in preparation). The progenitors of other intermediate-luminosity SNe II-P, therefore, have not 
yet been directly identified, although not without efforts in analyzing pre-SN data to do so in the recent past, such as for SN 1999em (Smartt et al. 2002).

The recent discovery of SN 2012aw in M95 (NGC 3351) by P. Fagotti on 2012 March 16.86 (UT dates are used throughout this paper), A. Dimai on 2012 March 16.84, and J. Skvarc on March 17.90 (reported in CBET 3054) has now afforded us with the best opportunity to do so. Indications of the nature of the SN during the plateau phase, which we will present in a forthcoming paper, are that it is similar to SN 1999em. A spectrum of the SN on March 17.77 by Munari (2012) showed a very hot, blue, essentially featureless continuum. A spectrum on March 18.77 by Siviero et al. (2012) also showed a blue, featureless continuum, and later spectra on March 19.85 and 19.92 exhibited the characteristics of a very young SN II-P, with the onset of broad lines having P-Cygni-like profiles. The object was also discovered by the Palomar Transient Factory (PTF; Law et al. 2009; Rau et al. 2009) and given the name PTF12bvh. It was first detected at $R \approx 14.2 \mathrm{mag}$ in an image obtained with the PTF camera (Rahmer et al. 2008) on the Palomar Oschin Schmidt 48 in (1.2 m) telescope on March 16.70. The SN was not visible up to and including March 14.75 to a limiting magnitude $R \gtrsim 22(3 \sigma)$, providing a tight constraint on the explosion epoch (Poznanski et al. 2012a originally reported $R \geqslant 20.7 \mathrm{mag}$, but this has since been revised to the fainter limit). The SN shows indications of interaction with circumstellar matter, through detection in the radio (Yadav et al. 2012; Stockdale et al. 2012) and X-ray (Immler \& Brown 2012) bands. It also exhibits signs, although preliminary, of possibly unusual polarization of the SN ejecta (Leonard et al. 2012).

The probable progenitor of SN 2012aw/PTF12bvh was first detected in HST images by Elias-Rosa et al. (2012) and subsequently by Fraser et al. (2012a). The apparently red color of the star indicated that it was most likely an RSG. Here we present photometry of the star and discuss its nature, including its likely initial mass. An analysis of the progenitor has also been conducted by Fraser et al. (2012b). In their study, Fraser et al. conclude that the RSG was observed through considerable visual extinction $\left(A_{V}>1.2 \mathrm{mag}\right)$, and their estimates of the star's luminosity and initial mass span a fairly large range, $10^{5.0}-10^{5.6} L_{\odot}$ and $14-26 M_{\odot}$, respectively.

For the distance to the $\mathrm{SN}$, we adopt the reddening- and metallicity-corrected distance modulus $\mu_{0}=30.00 \pm 0.09 \mathrm{mag}$ for M95 determined by Freedman et al. (2001).

\section{PROGENITOR OBSERVATIONS}

The SN site was imaged by HST in F439W, F555W, and F814W with the Wide-Field Planetary Camera 2 (WFPC2) between 1994 December and 1995 January, as part of the HST Key Project to use Cepheid-based distances to measure the value of the Hubble constant (GO-5397; PI: J. Mould). The Hubble Legacy Archive (HLA) project has subsequently combined the individual WFPC2 exposures, using the task MultiDrizzle (Koekemoer et al. 2003; see also Fruchter \& Hook 2002), into deep image mosaics in F555W and F814W. The total exposure times are 30,130 s and $9830 \mathrm{~s}$ in these two bands, respectively. The SN progenitor at F814W is shown in Figure 1. The HLA did not produce mosaics at $\mathrm{F} 439 \mathrm{~W}$; the total exposure time is $5000 \mathrm{~s}$ for these four images. The SN site was also imaged in F555W on 1995 December 4 by program GO-5972 (PI: J. Mould) for $2000 \mathrm{~s}$ total, and in F336W and F658N on 2009 January 18 by program GO-11966 (PI: M. Regan) for $4400 \mathrm{~s}$ and $1800 \mathrm{~s}$ total, respectively.
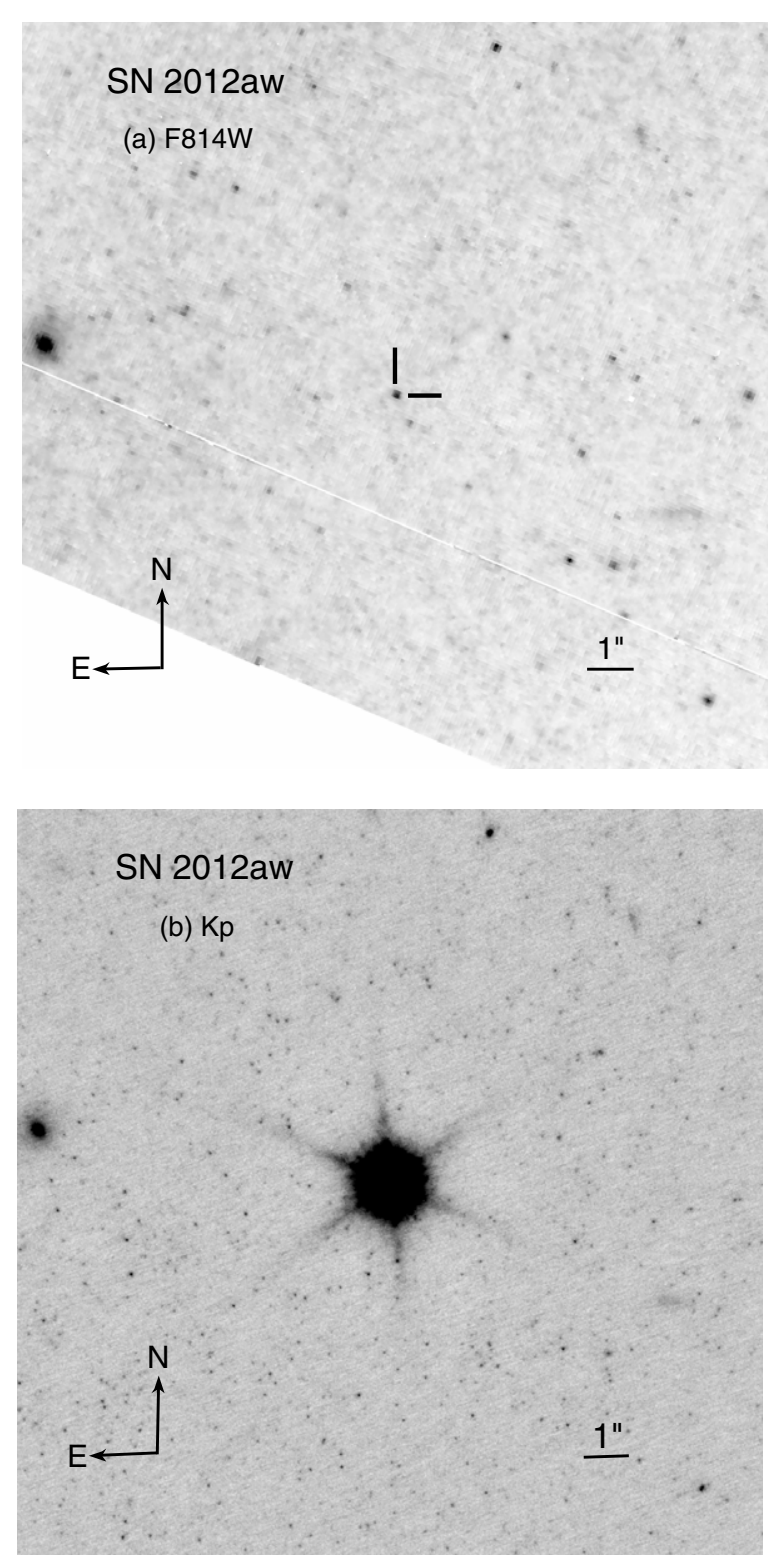

Figure 1. (a) A portion of the archival HST F814W image of M95 from 1994/1995; the star detected at the precise location of SN 2012aw is indicated by tick marks. The SN site is very near to the edge of the image mosaic. (b) A portion of the $K_{p}$-band AO image obtained using NIRC2 on the Keck-II telescope on 2012 March 27.

Images of the SN were obtained on March 21.31 in the $i^{\prime}$ band in sets of exposures with times of $5 \mathrm{~s}$ and $10 \mathrm{~s}$, under very good observing conditions $\left(\sim 0^{\prime \prime} .8\right)$, using the MegaCam on the $3.6 \mathrm{~m}$ Canada-France-Hawaii Telescope (CFHT), to initially locate the SN position in the pre-SN archival HST images (see below). From these images we also measured an absolute position for the SN of $\alpha(\mathrm{J} 2000)=10^{\mathrm{h}} 43^{\mathrm{m}} 53.73, \delta(\mathrm{J} 2000)=+11^{\circ} 40^{\prime} 17^{\prime \prime} .9$ $( \pm 0$ '. 11 root mean square), relative to 12 stars in the field from the USNO B1.0 Catalog (Monet et al. 2003). To further refine the SN position, relative to the HST images, we obtained three sets of dithered Near-Infrared Camera 2 (NIRC2) images of the $\mathrm{SN}$ in the $K_{p}$ band (with exposure times $1.5,3$, and $10 \mathrm{~s}$, respectively), using adaptive optics (AO) and the $10 \mathrm{~m}$ Keck II telescope on March 27.42. The SN at $K_{p}$ is shown in Figure 1. Since the SN was quite bright at the time of these observations, it was possible to perform the $\mathrm{AO}$ using it as a natural guide star, so that the laser guide star was not necessary. 
Table 1

Photometry of the SN 2012aw Progenitor ${ }^{\mathrm{a}}$

\begin{tabular}{lc}
\hline \hline Band & Magnitude \\
\hline F336W & $\gtrsim 22.4^{\mathrm{b}}$ \\
F439W & $\gtrsim 25.9^{\mathrm{b}}$ \\
F555W & $26.49(07)$ \\
$V$ & $26.59(07)$ \\
F814W & $23.39(02)$ \\
$I$ & $23.44(02)$ \\
$J$ & $21.02(03)$ \\
$K_{S}$ & $19.47(19)$ \\
\hline
\end{tabular}

Notes.

${ }^{\mathrm{a}}$ Uncertainties $(1 \sigma)$ are given in parentheses, in units of $0.01 \mathrm{mag}$.

$\mathrm{b}$ These are $3 \sigma$ upper limits.

Using the CFHT images, we were able to locate a candidate progenitor star for the SN in the HLA F814W image mosaic, with an error circle radius of $\sim 0.6$ pixels ( $0{ }^{\prime} .03$ for these drizzled mosaics), the results of which were first presented by Elias-Rosa et al. (2012). For the Keck AO imaging, from a co-addition of 40 individual $3 \mathrm{~s}$ frames (no geometric distortion correction was applied prior to the co-addition) in which the SN is only mildly saturated, we used 16 stars in common between this coadded mosaic and the HLA F814W mosaic with the IRAF task geomap to register the two mosaics with an uncertainty in the image transformation of $\Delta x=0.204$ and $\Delta y=0.591$ HLA mosaic pixels ( $0^{\prime \prime} 010$ and $0^{\prime \prime} 030$, respectively). We find that the locations of the $\mathrm{SN}$ and progenitor candidate are consistent to within the $1 \sigma$ statistical uncertainty of our alignment procedure. We conclude that we have confirmed the candidate star seen in the WFPC2 mosaics as the probable progenitor of SN 2012aw. This is the same star that was identified by Fraser et al. (2012a, 2012b).

Using Dolphot v2.0, as applied to WFPC2 data (Dolphin 2000a, 2000b), we measured the apparent brightness of the progenitor from the ensemble of individual images in both F555W and F814W from GO-5397. For F555W we ultimately input into Dolphot only the images that produced uncertainties in the photometry of $\lesssim 0.28 \mathrm{mag}$; in this band, the star is detected at only $\sim 4 \sigma$ per individual exposure in the first place. We also omitted one F814W exposure that was appreciably noisier than all the others (its exposure time was only $230 \mathrm{~s}$, compared to $1000-1500 \mathrm{~s}$ for the other exposures in this band). We used one of the remaining $\mathrm{F} 814 \mathrm{~W}$ exposures (the star is generally detected at $\sim 22 \sigma$ per exposure) as the astrometric reference image in Dolphot. The output from Dolphot automatically includes the transformation from flight-system F555W and F814W to the corresponding Johnson-Cousins (Bessell 1990) magnitudes in $V$ and $I_{C}$. (We refer to $I_{C}$ as $I$ hereafter.) We find that $m_{\mathrm{F} 555 \mathrm{~W}}=26.49 \pm 0.07$ and $m_{\mathrm{F} 814 \mathrm{~W}}=23.39 \pm 0.02 \mathrm{mag}$. These flight-system magnitudes transform to $V=26.59$ and $I=$ 23.44 mag. Although our measurement at F814W is identical to that obtained by Fraser et al. (2012b), our measurement at F555W is brighter by $>1 \sigma$ than their value. For F336W and F439W the star was not detected by Dolphot to $m_{\mathrm{F} 336 \mathrm{~W}} \gtrsim 22.4$ and $m_{\mathrm{F} 439 \mathrm{~W}} \gtrsim 25.9 \mathrm{mag}$ (both $3 \sigma$ ). The measurements of the progenitor's apparent brightness (or limits to the apparent brightness) are summarized in Table 1.

As Fraser et al. (2012b) discuss, there are also archival ground-based near-infrared images that contain the SN progenitor, namely, those obtained at the European Southern
Observatory, with the Infrared Spectrometer and Array Camera (ISAAC) on the $8.2 \mathrm{~m}$ Very Large Telescope Unit Telescope 1 on 2000 March 26 and 27 (PI: F. Bresolin), and with the Son of ISAAC (SOFI) on the $3.6 \mathrm{~m}$ New Technology Telescope on 2006 March 24 (PI: J. Ascenso). The ISAAC images we used were in the $J_{s}$ band, with $30 \mathrm{~s}$ individual frame times and four subintegrations (co-additions) in memory, and the SOFI images were in the $K_{s}$ band, with $8 \mathrm{~s}$ frame times and 15 subintegrations. The progenitor site is also in $K_{s}$ images of the host galaxy, obtained as commissioning data on 2000 March 23 with the Isaac Newton Group Red Imaging Device (INGRID) on the $4.2 \mathrm{~m}$ William Herschel Telescope. The results of that imaging have been reported by Knapen et al. (2003).

Fraser et al. (2012b) calibrated their photometric analysis of the ISAAC and SOFI data sets using Two Micron All Sky Survey (2MASS) stars in the images. The problem with this approach is that these stars are all near the photometric limit of the 2MASS survey, $J \approx 16.4$ mag for the ISAAC images and $K_{s} \approx 14.7-15.5$ mag for the SOFI images. At this limit we expect the photometry to be far less reliable, as described in the 2MASS Explanatory Supplement, ${ }^{7}$ and therefore calibration of the ISAAC and SOFI image data using these stars could suffer from systematic effects, leading to a possible error in the final apparent magnitudes for the progenitor.

We therefore reanalyzed the ISAAC and INGRID images, including the original calibration data for those observations, and reanalyzed the SOFI images, applying the calibration at $K_{s}$ from the INGRID observations to those with SOFI, employing five well-detected stars in common between the two data sets. This calibration should be valid, since the bandpasses of both the INGRID and SOFI $K_{s}$ filters are quite similar. The data reduction of the raw frames followed standard procedures for near-infrared imaging. For the ISAAC, SOFI, and INGRID data we first corrected the individual images for the instrumental response with a combined, normalized flat frame obtained in each band. For the ISAAC observations, twilight-sky frames had been obtained for this purpose; for SOFI observations, dome flats were obtained; and for the INGRID observations, we produced a flat from a median-filtered combination of the off-source sky frames. We then subtracted from the on-source images a median-filtered combination of the sky frames. For each band a reference frame was chosen, all other frames were shifted in pixel space relative to the reference, and the shifted frames were co-added, together with the reference frame, to produce a single image mosaic. For the March 26 ISAAC data we co-added 16 individual $J_{s}$ frames, and for the March 27 data we co-added 17 frames. For the SOFI $K_{s}$ imaging we co-added all five on-source frames. For the INGRID imaging we co-added nine of the $20 \mathrm{~s}$ frames that contained the progenitor site.

We extracted photometry from all of the image mosaics using point-spread function fitting in IRAF/DAOPHOT (Stetson 1987). The progenitor star was detected in the $J_{s}$ mosaics from the two ISAAC nights at a signal-to-noise ratio $(\mathrm{S} / \mathrm{N})$ of $\sim 25-35$, whereas the star was detected in both the SOFI and INGRID mosaics at only $\mathrm{S} / \mathrm{N} \approx 4$. We measured aperture photometry using IRAF for the calibration-star observations obtained for both the ISAAC and INGRID runs. For ISAAC these consisted of various Persson et al. (1998) standard stars observed on both nights. For both 2000 March 26 and 27 we found solutions with airmass corrections of $0.100-0.105 \mathrm{mag}$ airmass $^{-1}$, essentially the canonical correction at $J$ (Persson

\footnotetext{
7 http://www.ipac.caltech.edu/2mass/releases/allsky/doc/explsup.html.
} 
et al. 1998; the rms uncertainty in the solution was 0.002 mag for March 28 and a slightly higher 0.009 mag for March 27). We therefore measured $J=21.03 \pm 0.04$ mag on March 26 and $J=21.01 \pm 0.03 \mathrm{mag}$ on March 27 for the progenitor. We note that, although the images are in $J_{s}$, while the photometric standard magnitudes are in the Las Campanas Observatory (LCO) $J_{\text {LCO }}$ band (Persson et al. 1998), we analyzed the synthetic photometry of three of these standard stars (P041-C, P177-D, and P330-E), for which there are also HST calibration spectra, and the magnitudes for these stars are only about 0.02 mag brighter through $J_{s}$ than $J_{\mathrm{LCO}}$; we also find that for the likely spectral energy distribution (SED) of the probable progenitor star (see below), the progenitor itself would be about 0.05 mag brighter in $J_{s}$ than in $J_{\mathrm{LCO}}$. (We hereafter refer to $J_{\mathrm{LCO}}$ merely as $J$.) We have added the latter difference as an additional uncertainty in quadrature with the uncertainty in the photometric measurement. The uncertainty-weighted mean of the two measurements is $J=21.02 \pm 0.03 \mathrm{mag}$.

For the INGRID calibration, two United Kingdom Infrared Telescope faint standards (Leggett et al. 2006), FS14 and FS21, had each been observed at the low airmasses that bracketed the observations of M95. We obtained aperture photometry of these two stars and calibrated the faint DAOPHOT photometry of the progenitor star accordingly. We note that the $K_{s}$ bandpass used for these observations is in the Mauna Kea Observatory (MKO) system (Simons \& Tokunaga 2002; Tokunaga et al. 2002). The star's brightness in this band is then $K_{s}=19.52 \pm 0.27 \mathrm{mag}$. Applying the calibration of the INGRID data to the SOFI image mosaic, as previously described, we obtain $K_{s}=19.42 \pm$ $0.29 \mathrm{mag}$ for the progenitor. The uncertainty-weighted mean of these two measurements is $K_{s}=19.47 \pm 0.19 \mathrm{mag}$.

We summarize the measurements of the progenitor's nearinfrared brightness in Table 1. Although, in the end, our measurements at $J$ and $K_{s}$ agree with those obtained by Fraser et al. (2012b) to within the uncertainties in their photometry, we were able to achieve a higher precision in our measurements and were able to reduce the uncertainties substantially through the standard-star calibrations.

\section{THE NATURE OF THE PROGENITOR STAR}

Unlike the lower-luminosity RSGs that are progenitors of low-luminosity SNe II-P, such as SN 2008bk (Mattila et al. 2008; Van Dyk et al. 2012), Massey et al. (2005) pointed out that we would expect higher-luminosity RSGs to lose mass at a significantly higher rate, and that this mass loss results in circumstellar shells where dust tends to form. We already have an indication that this RSG was of high luminosity, from its $K$-band brightness; even neglecting extinction (which, in the first place, at $K$ is typically nearly a factor of 10 less than at $V)$ and assuming a bolometric correction $\mathrm{BC}_{K} \approx 3 \mathrm{mag}$, the bolometric magnitude for the star would be $M_{\mathrm{bol}} \approx-7.8$.

What is striking about this progenitor detection is that the star is sufficiently luminous to be easily visible in both the HST F814W image and the ground-based near-infrared images. Another interesting facet is the relative isolation of the progenitor in the host galaxy; it is apparently far from any noticeable cluster, OB association, or H II region. Most likely, this isolation largely contributes to the detectability of the star at the distance of the host galaxy, since the star's environment is uncrowded. Furthermore, it is tempting to speculate that, like the famous Galactic RSG Betelgeuse ( $\alpha$ Orionis; e.g., NoriegaCrespo et al. 1997), the SN 2012aw progenitor could also have been a stellar runaway.

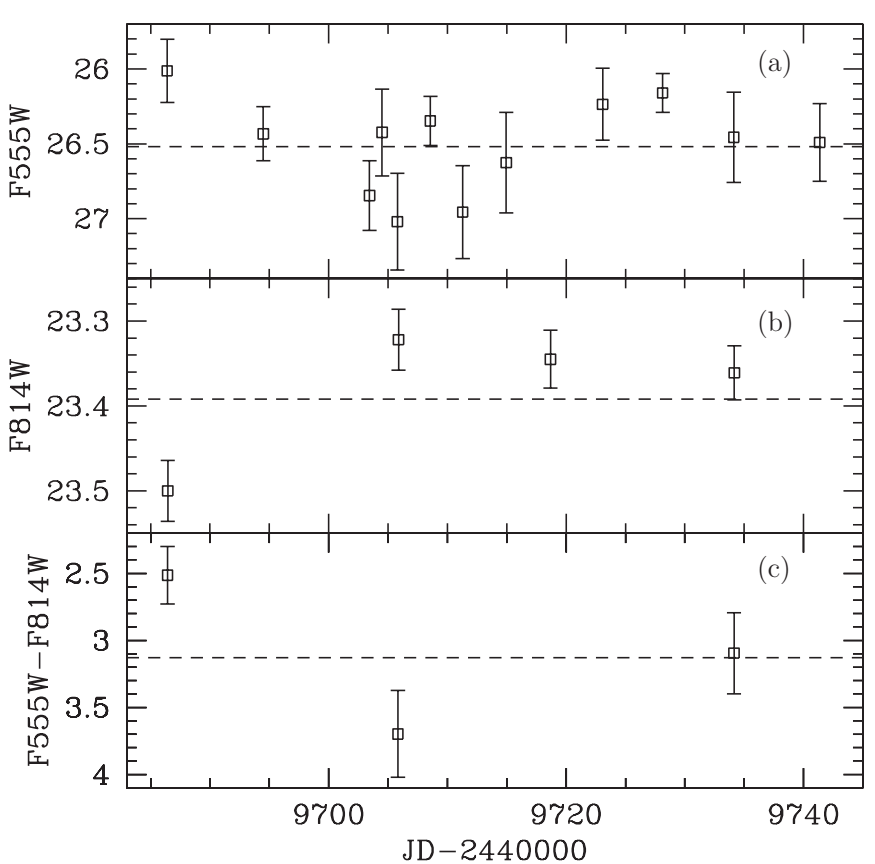

Figure 2. Photometry (mag) of the SN 2012aw progenitor measured with Dolphot from the individual $H S T$ /WFPC2 observations from program GO-5397 in the (a) F555W and (b) F814W bands. Observations obtained on the same date in a given band have been averaged, weighted by the uncertainties in the individual measurements. Panel (c) shows the F555W-F814W color (mag). The dashed lines in all three panels arise from the uncertainty-weighted means of all measurements in each band, as returned by Dolphot.

\subsection{Variability of the Progenitor}

It is known that RSGs can be semiregular or irregular variable stars. Kiss et al. (2006) analyzed the optical variability of 48 Galactic RSGs and found evidence for two modes of variability in 18 of the stars (e.g., $\alpha$ Ori), one mode with periods of a few hundred days and one of a few thousand days. Concern, therefore, should exist whether the apparent brightnesses that we have measured in the various bands, especially the ensemble from the HST images, are representative of the star's actual brightness. Whereas variability at $V$ can be considerable, up to a magnitude or more, RSGs show essentially no variability at $K$ (e.g., Levesque et al. 2007).

The HST data for M95 were obtained over a number of epochs spanning $\sim 65$ days (those data were originally taken to discover and measure the periods of Cepheids in the host galaxy), so we can analyze the multi-epoch photometry for any indication of short-period variability of the progenitor in 1994/1995, 17-18 yr prior to the explosion. In Figure 2, we show the individual measurements with Dolphot from the HST/WFPC2 images in both F555W and F814W. For HST observations that were made on essentially the same Julian date, we have computed an uncertainty-weighted mean value for the star's brightness and show those in the figure, rather than the two individual measurements for a given date. This was particularly the case for the F814W measurements. We also show estimates of the color from three epochs.

Clearly excursions of the measurements exist, relative to the mean brightness for the star in each band, with total apparent variations of $\Delta m_{\mathrm{F} 555 \mathrm{~W}} \approx 1 \mathrm{mag}$ and $\Delta m_{\mathrm{F} 814 \mathrm{~W}} \approx 0.18 \mathrm{mag}$. We must determine the significance of these variations. For that we have computed the reduced $\chi^{2}$ statistic in each band and find $\chi_{\text {red }}^{2}=1.92$ for F555W and $\chi_{\text {red }}^{2}=5.16$ for F814W. We can also assess the $p$-value of these two statistics; a $p$-value of 0.05 
or less is usually regarded as statistically significant (i.e., the observed deviation from the mean is significant). For F555W we find $p=0.033$, and for F814W, $p=0.001$. We therefore consider it likely that the variability we observe is real. We note that Fraser et al. (2012b) seemingly dismiss the possibility of variability in either band.

The mean color is already $\sim 0.8$ mag redder in $V-I$ than the color of an early-M supergiant in the absence of dust. The variation in the star's color is $V-I \approx 0.6 \mathrm{mag}$, relative to the mean. We clearly do not have spectra of the star, so we cannot determine whether the color variation represents true changes in the star's effective temperature (e.g., Massey et al. 2007), or merely variations in the line-of-sight dust. If the former, the color variations would correspond to variations in the mean effective temperature of $\sim 300 \mathrm{~K}$.

Although the progenitor shows considerable variability over the $\sim 65$ days at $\sim V$, which would be consistent with variations in the dust content of the star's circumstellar material (e.g., Massey et al. 2005, 2007), and more limited variability at $\sim I$, we will assume that the mean brightness at both $V$ and $I$ over this timescale is representative of the star's brightness over far longer timescales, at least through the year 2000, when the $J$ and $K_{s}$ brightness were sampled. We would expect the $K_{s}$ brightness to have been relatively constant. We have no insight into the level of variability at $J$, but we might expect it to be considerably less than at $I$. We therefore assume that the progenitor's observed brightness in all of these bands fairly represents the actual SED of the star.

\subsection{Metallicity in the SN Environment}

Assuming that the oxygen abundance can serve as a proxy for the overall metallicity in the host galaxy, we estimated the metallicity at the SN site based on the gradient in the disk of M95 of the O abundance measured by Moustakas et al. (2010). We deprojected the MegaCam image, assuming the values for the position angle and inclination for M95 from Moustakas et al., and measured the radial offset of the SN position from the nuclear position. For a plate scale of 0.185 pixel $^{-1}$, we find that this offset is $\rho=162^{\prime \prime}$.02, or 2.70. Again, assuming the radial offset at $25 \mathrm{~B} \mathrm{mag} \operatorname{arcsec}^{-2}$ for M95 from Moustakas et al. (2010), i.e., $\rho_{25}=3$ '.71, we then calculate that $\rho / \rho_{25}=0.73$. At this scaled nuclear offset, assuming the abundance in Moustakas et al. derived from the strong-line index calibration by Pilyugin $\&$ Thuan $(2005)$, we find that $12+\log (\mathrm{O} / \mathrm{H}) \approx 8.5$ in the $\mathrm{SN}$ environment. Given that the solar value is $12+\log (\mathrm{O} / \mathrm{H})=$ $8.66 \pm 0.05$ (Asplund et al. 2005), we infer that the metallicity at the SN site is only slightly subsolar and likely still consistent with solar, given the uncertainties. We therefore analyze our results assuming solar metallicity.

\subsection{Properties of the Star}

From the relatively high apparent brightness at $K_{s}$ for the star, compared to the relative faintness at $V$, we realized that the reddening to the progenitor had to be high, likely due to circumstellar dust. Massey et al. (2005) demonstrated that the effective total-to-selective ratio of absorption, $R_{V}^{\prime}$, for the dust around Galactic RSGs should differ considerably from the $R_{V}=3.1$ typical of the diffuse interstellar medium (ISM; e.g., Cardelli et al. 1989). This may imply a difference, for example, in the grain-size distribution in these shells. They found from their sample of RSGs that $R_{V}^{\prime}=4.1+0.1 E(B-V)-0.2 \log g$, where $\log g$ is the surface gravity of the RSG and has values of between -0.5 and 0.5 (when $g$ is expressed in cgs units).
We compared the observed SED for the progenitor to a model SED synthesized using STSDAS/SYNPHOT within IRAF from the MARCS model stellar atmospheres for RSGs at solar metallicity (Gustafsson et al. 2008) with $T_{\text {eff }}$ in the range 3400-3800 K (essentially, spectral types of M5 through late K; Levesque et al. 2005), in steps of $100 \mathrm{~K}$, and surface gravities $\log g=-0.5,0.0$, and +0.5 . The models are for $15 M_{\odot}$ stars, assuming spherical geometry and a microturbulence velocity of $5 \mathrm{~km} \mathrm{~s}^{-1}$. The filter response function at $J$ in the LCO system is taken from Persson et al. (1998), and the function for $K_{S}$ in the MKO system is from the online INGRID instrument page. ${ }^{8}$ We allowed $R_{V}^{\prime}$ to vary from the typical value of 3.1 to larger values and computed a range of $A_{V}$ from 1.4 to $4.2 \mathrm{mag}$ for each $R_{V}^{\prime}$, following Cardelli et al. (1989). We were able to eliminate the $\log g=+0.5$ models outright, since none of these were able to reproduce the observed SED at any value of $R_{V}^{\prime}, A_{V}$, and $T_{\text {eff }}$. For all of the models at the other two surface gravities, we found that only the $T_{\text {eff }}=3600 \mathrm{~K}$ models were allowed within the uncertainties in the photometry. Furthermore, we found that for the $\log g=-0.5$ models, a range in $R_{V}^{\prime}$ from 4.0 to 4.7 was allowed, although the range in $A_{V}$ was constrained to $3.05-3.10 \mathrm{mag}$. For the $\log g=0.0$ models, $R_{V}^{\prime}$ could range from 4.1 to 4.6 , and again, the extinction was constrained to $A_{V}=3.10-3.15 \mathrm{mag}$.

The total $A_{V}$ in this case can be considered an excess extinction, due to circumstellar dust, together with the lineof-sight extinction to the star, which we assume to be the total extinction to the SN (see Section 3.5). Since we have shown that the star was variable, possibly due to variations in the amount of circumstellar dust, this total $A_{V}$ is essentially a time-averaged value over the duration of both the $H S T$ and ground-based observations.

The average values of $R_{V}^{\prime}$ and $A_{V}$ (in mag) for the models at both surface gravities are 4.35 and 3.10 , respectively. We show in Figure 3 a representative model at $T_{\text {eff }}=3600 \mathrm{~K}$ with these values of $R_{V}^{\prime}$ and $A_{V}$. The tightest constraint on the model SEDs comes from the $I$-band measurement; the large uncertainty at $K_{s}$, on the other hand, is not as constraining. From Levesque et al. (2005), this effective temperature would correspond to spectral type M3.

Following Levesque et al. (2005) and Bessell et al. (1998), we computed the bolometric corrections at $V, I$, and $K_{s}$ from the MARCS RSG stellar atmosphere models at $T_{\text {eff }}=3600 \mathrm{~K}$ and at $\log g=-0.5$ and $\log g=0.0$. We found $\mathrm{BC}_{V}=-1.79$, $\mathrm{BC}_{I}=0.33$, and $\mathrm{BC}_{K_{s}}=2.79$ mag for $\log g=-0.5$, and $\mathrm{BC}_{V}=-1.78, \mathrm{BC}_{I}=0.32$, and $\mathrm{BC}_{K_{s}}=2.80$ mag for $\log g=$ 0.0. Assuming that $R_{V}^{\prime}=4.35$ and $A_{V}=3.10 \mathrm{mag}$, we find that $M_{\text {bol }}=-8.29 \pm 0.12,-8.29 \pm 0.11$, and $-8.13 \pm 0.22$ mag from $V, I$, and $K_{s}$, respectively, adding in quadrature the uncertainties in the photometric measurements, in the inferred extinction (0.05 mag), in the bolometric corrections $(0.01 \mathrm{mag})$, and in the host-galaxy distance modulus. It is very satisfying that our estimates of $M_{\text {bol }}$ are exactly the same at $V$ and $I$, which gives us confidence that the values for $R_{V}^{\prime}, A_{V}, T_{\text {eff }}$, and $\log g$, taken together, are all consistent. It also allows us to neglect $M_{\mathrm{bol}}$ obtained from the far less certain $K_{s}$ measurement, although the value of $M_{\text {bol }}$ from this band certainly agrees with those from the other two bands, to within the uncertainties. We therefore adopt the uncertainty-weighted mean, $M_{\text {bol }}=-8.29 \pm 0.08 \mathrm{mag}$, from $V$ and $I$. We conservatively adopt an uncertainty of $\pm 100 \mathrm{~K}$ in $T_{\text {eff }}$, although the star's observed SED appears to

\footnotetext{
8 http://www.ing.iac.es/Astronomy/instruments/ingrid/ingrid_filters.html.
} 


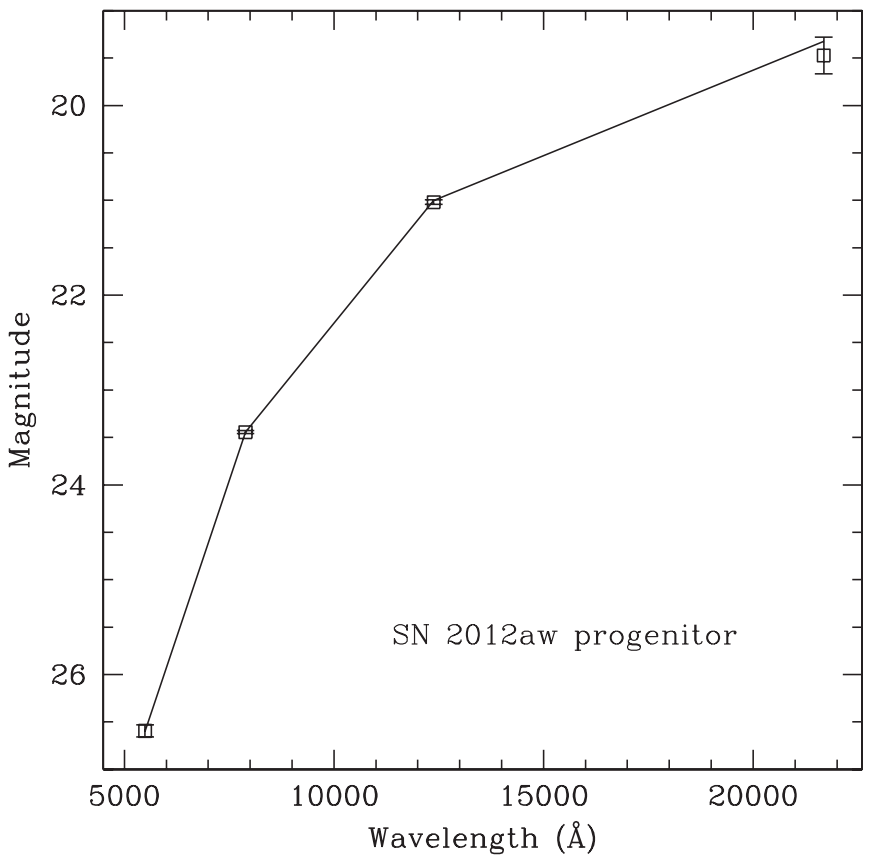

Figure 3. Observed SED for the probable SN 2012aw progenitor. See Table 1. Also shown for comparison is an example SED computed via synthetic photometry of a MARCS model RSG stellar atmosphere (Gustafsson et al. 2008) with surface gravity $\log g=-0.5$, effective temperature $T_{\text {eff }}=3600 \mathrm{~K}$, and extinction $A_{V}=3.10 \mathrm{mag}$ (solid line), assuming an effective total-to-selective ratio of absorption, $R_{V}^{\prime}=4.35$. Although a surface gravity $\log g=0.0$ and a limited range in $R_{V}^{\prime}$ are also allowed, both $A_{V}$ and $T_{\text {eff }}$ are constrained by the observations; see the text. The model SED has been normalized at $I$, the band that provides the tightest photometric constraint.

tightly constrain the effective temperature of the star to less than this uncertainty. Assuming $M_{\text {bol }}(\odot)=4.74 \mathrm{mag}$, this corresponds to a bolometric luminosity relative to the Sun of $\log \left(L_{\text {bol }} / L_{\odot}\right)=5.21 \pm 0.03$.

In Figure 4, we show a Hertzsprung-Russell diagram with the locus of the SN 2012aw progenitor. For comparison, we also illustrate the massive-star evolutionary tracks at solar metallicity from Ekström et al. (2012) for stars with initial rotation that is $40 \%$ of the critical rotation, at initial masses $M_{\text {ini }}=15$ and $20 M_{\odot}$. The star is clearly more luminous than the RSG terminus of the $M_{\text {ini }}=15 M_{\odot}$ model, although the star's effective temperature is consistent with the value for the terminus of this model. The $20 M_{\odot}$ model, however, terminates at a higher luminosity and far hotter $T_{\text {eff }}$. The "red loop" of that track does approach the star's locus; however, based on the behavior of that track, we do not expect a star with this initial mass prematurely to reach its endpoint at this luminosity and cooler effective temperature along the loop. We can infer, therefore, that the progenitor's initial mass was in the range $15 \lesssim M_{\text {ini }}\left(M_{\odot}\right)<20$. Ekström et al. (2012) do not provide model tracks between $M_{\text {ini }}=15$ and $20 M_{\odot}$, so it is unclear at what luminosity and effective temperature stars with initial masses within this range would reach their termini. However, by eye from the figure, we can interpolate that the star's locus could well be consistent with the endpoints of a putative 17 or $18 M_{\odot}$ track. Clearly, we require the actual model track to be more precise about this initial mass assignment.

At the adopted effective temperature and luminosity, the star had an effective radius $R=1040 \pm 100 R_{\odot}$. From the Ekström et al. (2012) $M_{\text {ini }}=15 M_{\odot}$ evolutionary track, the final mass is $M_{\mathrm{fin}}=11.1 M_{\odot}$, so the surface gravity would then be

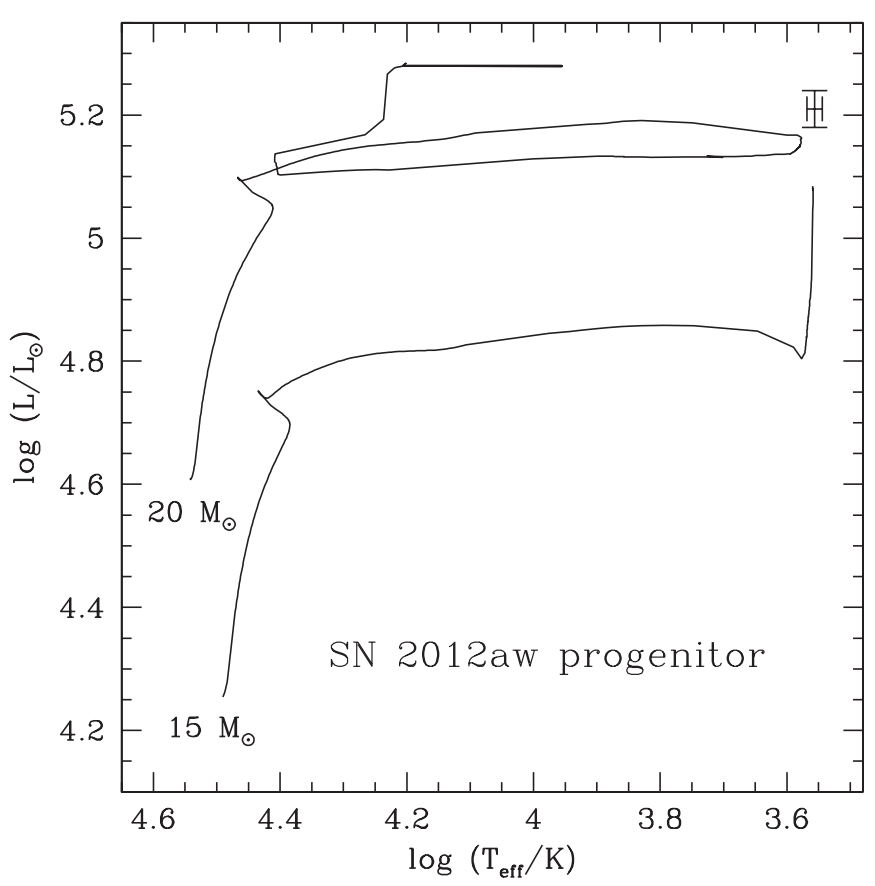

Figure 4. Hertzsprung-Russell diagram showing the locus of the SN 2012aw progenitor. For comparison we also indicate massive-star evolutionary tracks at solar metallicity, which include initial rotation on the main sequence that is 0.4 times the critical rotation velocity, from Ekström et al. (2012) for $M_{\text {ini }}=15$ and $20 M_{\odot}$.

$\log g \approx-0.5$. From the adopted $A_{V}$ and $R_{V}^{\prime}$, the reddening $E(B-V)=0.71 \mathrm{mag}$. We then would expect, from the relation from Massey et al. (2005) between $R_{V}^{\prime}, E(B-V)$, and $\log g$, that $R_{V}^{\prime} \approx 4.27$. This indicates that there is consistency among these three parameters taken together, and this crosscheck further provides us with confidence in our estimates of the star's properties.

We note that the main difference between the analysis we have presented here and that presented by Fraser et al. (2012b) - namely, that we are able to better constrain the effective temperature, luminosity, and, therefore, the initial mass estimate for the progenitor-stems not only from the differences in the photometry between the two studies (we found that the star is brighter in $V$, and we were also able to reduce the uncertainties at $J$ and $K_{s}$ ), but also from our assumption that $R_{V}$ for the RSG progenitor was different from the typical value of 3.1 for the diffuse ISM. One item to also note is that the evolutionary tracks (without rotation; Eldridge et al. 2008) employed by Fraser et al. (2012b) tend to terminate at significantly cooler effective temperatures than the Ekström et al. (2012) tracks that we have used. The key, ultimately, was connecting the inferred properties of the SN 2012aw progenitor, based on our measurements, to those of the Galactic RSGs of similar luminosity and mass (Massey et al. 2005).

\subsection{The Dust around the Progenitor}

As Massey et al. (2005) discussed, we can estimate the mass of the dust and duration of dust production that is responsible for the excess extinction that we infer for the RSG progenitor. Massey et al. assume a thin-shell approximation for the excess extinction, $\Delta A_{V}=\Delta R\left(3.2 \times 10^{3}\right) M_{d} /\left(4 \pi R^{2} \Delta R\right)$, where $R$ is the stellar radius (in $\mathrm{m}$ ), $\Delta R$ is the extent of the thin dust layer or shell (or the path length through the dust) above the stellar surface, and $M_{d}$ is the dust mass (in $\mathrm{kg}$ ). The circumstellar 


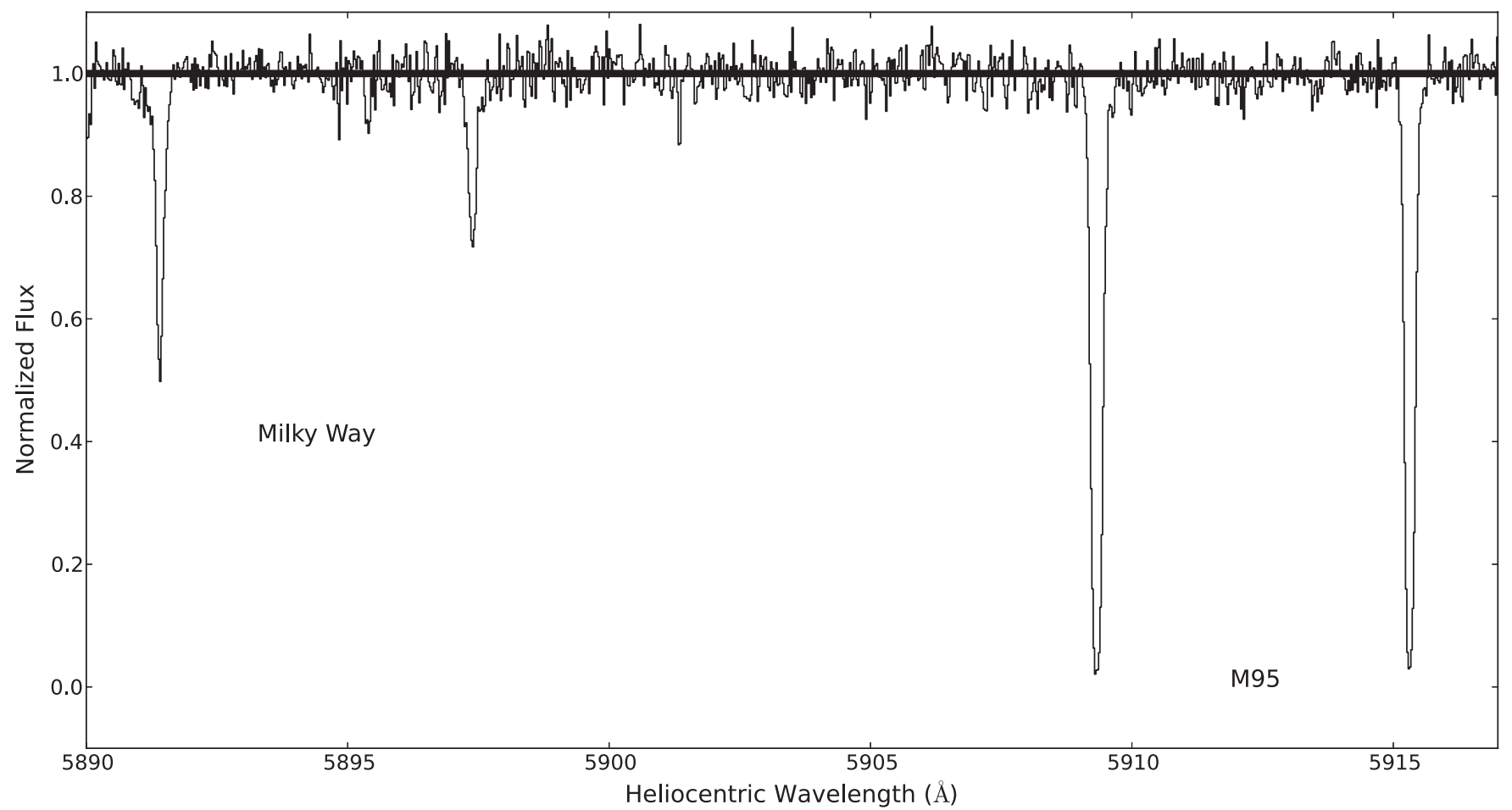

Figure 5. Spectrum of SN 2012aw obtained on 2012 April 10.29 with HIRESr on the $10 \mathrm{~m}$ Keck I telescope, centered on the Na I D absorption feature. Both feature components D1 $\lambda 5896$ and D2 $\lambda 5890$ are clearly detected from both the Galactic foreground (labeled as "Milky Way") and the SN host galaxy (labeled as "M95"). The features due to the host may be partially saturated.

matter presumably extended several stellar radii above the star's surface, as in the case of $\alpha$ Ori, and the overall mass loss could well have been driven by convection in the envelope (Josselin \& Plez 2007; Chiavassa et al. 2010). It is in the last $\sim 1400 \mathrm{yr}$ of the RSG phase, as seen in the $15 M_{\odot}$ model from Ekström et al. (2012), that the star's luminosity, radius, and total mass loss particularly increase. From Figure 4 of Massey et al. (2005), we see that the inferred $\Delta A_{V} \approx 3 \mathrm{mag}$ (we will show in Section 3.5 that the interstellar extinction, for $R_{V}=3.1$, is likely only $A_{V} \approx 0.2 \mathrm{mag}$ ) is entirely consistent with the $M_{\text {bol }}$ of the star. The dust production rate, $\dot{M}_{d}$, corresponding to this luminosity is $\sim 10^{-8.44} M_{\odot} \mathrm{yr}^{-1}$. From the relation above, the value of $M_{d}$ is $\sim 6.1 \times 10^{21} \mathrm{~kg}$. Comparing this to $\dot{M}_{d} \Delta t$, we find that a dust-producing episode of $\Delta t \approx 1 \mathrm{yr}$, at some point prior to 1994 (since this dust already existed by the time of the first $H S T /$ WFPC2 images), could account for $\Delta A_{V}$. Although quite a short interval of time, it is consistent with that inferred for episodic dust production in Galactic RSGs (Danchi et al. 1994; Bester et al. 1996).

\subsection{The Dust around the Supernova}

However brief was the dust production, as Fraser et al. (2012b) point out, the dust was far more quickly destroyed, likely by the $\mathrm{X}$-ray/UV flash within hours of core collapse. As also noted by Fraser et al. (2012b), such circumstellar dust destruction is not unprecedented for other SNe (e.g., Dwek et al. 2008; Wesson et al. 2010).

Poznanski et al. (2011) have found that the Na I D feature strength in low-resolution optical SN spectra is a poor indicator of the amount of extinction to the SN. However, Poznanski et al. (2012b) have established a well-calibrated relation between reddening and the equivalent width $(\mathrm{EW})$ of the $\mathrm{Na}$ I $\mathrm{D}$ doublet, D1 and D2, based on more than 100 high-resolution spectra of objects through a number of interstellar lines of sight. We have therefore measured the EW of the doublet, from both the Milky Way and host-galaxy components, as clearly detected in a high-resolution spectrum of SN 2012aw that we obtained with the High Resolution Echelle Spectrometer (Vogt et al. 1994) on the $10 \mathrm{~m}$ Keck I telescope in the red optics configuration ("HIRESr") on 2012 April 10.29. We used the C2 decker (i.e., the 0 .'86 slit), providing coverage of $\sim 3800-7300 \AA$ with a resolution of 50,000 . The portion of this spectrum, centered around the $\mathrm{NaI} \mathrm{D}$ feature, is shown in Figure 5. We find that for the Milky Way component, EW(D2 $\lambda 5891.41)=$ $94 \pm 8 \mathrm{~m} \AA$ and $\mathrm{EW}(\mathrm{D} 1 \lambda 5897.39)=56 \pm 9 \mathrm{~m} \AA$. For the component internal to M95, EW(D2 $\lambda 5909.33)=269 \pm 14$ $\mathrm{m} \AA$ and $\mathrm{EW}(\mathrm{D} 1 \lambda 5915.32)=231 \pm 11 \mathrm{m \AA}$. Uncertainties in the centroids of the absorption features are typically $\sim 0.05 \AA$. (These are all vacuum wavelengths, corrected to a heliocentric frame of reference.) The features due to M95, particularly, the D2 component and, to a lesser extent, the D1 component, may be slightly saturated.

From the Poznanski et al. (2012b) relations, $E(B-V)=$ $0.022 \pm 0.013 \mathrm{mag}$ from the Milky Way and $E(B-V)=$ $0.055 \pm 0.014 \mathrm{mag}$ for the host galaxy. (The uncertainties here are primarily from the systematic uncertainties in the relations; the measurement uncertainties are comparatively negligible.) These relations take into account saturation in the features, which is a smaller effect compared to the systematic uncertainties in the relations. Our reddening estimate is consistent with the Galactic foreground reddening estimate from Schlegel et al. (1998), $E(B-V)=0.028 \mathrm{mag}$. The total reddening indicated from the high-resolution $\mathrm{SN}$ spectrum, assuming $R_{V}=3.1$, is then $E(B-V)=0.077 \mathrm{mag}$, which is relatively low and comparable to the estimated $E(B-V)=0.1 \mathrm{mag}$ found for SN 1999em (Baron et al. 2000; Leonard et al. 2002; Elmhamdi et al. 2003). The visual extinction to the $\mathrm{SN}$ is then $A_{V}=0.24$ mag. Although Poznanski et al. (2009) found that 
for a sample of SNe II-P the best-fit $R_{V}^{\prime} \approx 1.7$, the relations from Poznanski et al. likely do not depend strongly on $R_{V}$, and therefore the reddening and extinction values we have estimated likely will not be significantly different if $R_{V} \neq 3.1$, especially local to the SN. Nonetheless, it is evident that the SN explosion must have destroyed much or all of the circumstellar dust around the progenitor, leaving only what is most likely interstellar lineof-sight extinction, which we measured from the high-resolution SN spectrum.

\section{CONCLUSIONS}

The probable progenitor of the intermediate-luminosity SN II-P 2012aw/PTF12bvh has been identified in archival HST optical and ground-based near-infrared images. Using the photometry extracted from those images, we have constructed an SED for the star, and we analyze the SED to show that the star was a luminous ( $\left.M_{\text {bol }}=-8.29 \mathrm{mag}\right)$ RSG with spectral type M3 $\left(T_{\text {eff }}=3600 \mathrm{~K}\right)$ and with substantial circumstellar dust up to $18 \mathrm{yr}$ before explosion. This dust was clearly destroyed by the explosion, since the current extinction to the $\mathrm{SN}$ is relatively low. Although the existing, state-of-the-art, theoretical stellar evolutionary tracks do not terminate at the locus of the star in the Hertzsprung-Russell diagram, we surmise that the star had an initial mass $\sim 17-18 M_{\odot}$.

This mass is essentially the same as the upper limit to the initial mass, $16.5 \pm 1.5 M_{\odot}$, that Smartt et al. (2009) derived for SN II-P progenitors. It is not evident whether this result for SN 2012aw can be generalized for other intermediateluminosity SNe II-P. The initial mass for the progenitor of SN 2004et is still a subject of debate (e.g., Li et al. 2005; Crockett et al. 2011). Additionally, the (7 $\sigma)$ upper limit to the detection of SN 1999em at $I=22.0 \mathrm{mag}$ from Smartt et al. (2002), at a distance of 11.7 Mpc (Leonard et al. 2003, which implies a luminosity limit only a factor of $\sim 1.4$ fainter than if SN $1999 \mathrm{em}$ were at $10.0 \mathrm{Mpc}$, as is SN 2012aw), precludes detection of an analog to the SN 2012aw progenitor. It therefore remains uncertain what is the upper limit on the initial mass of the RSGs that give rise to "normal," intermediate-luminosity SNe II-P. The detected, unusual progenitors of high-luminosity SNe II-P (e.g., SN 2008cn; Elias-Rosa et al. 2009) may provide some indication of this limit. The recent stellar evolutionary tracks from Ekström et al. (2012), as well as those with pulsationally driven superwinds by Yoon \& Cantiello (2010), demonstrate the role of rotation and mass loss in the late-stage evolution of stars with $M_{\text {ini }} \gtrsim 20 M_{\odot}$. Still to be investigated more fully are the influences of factors such as the metallicity and binarity (e.g., Smith et al. 2011). To verify the candidate SN 2012aw progenitor, we will need to return at very late times, when the SN has substantially faded, to see whether the dusty RSG has vanished.

We thank the referee for useful comments that helped improve this manuscript. This work is based in part on observations with the NASA/ESA Hubble Space Telescope, obtained at the Space Telescope Science Institute (STScI), which is operated by AURA, Inc., under NASA contract NAS5-26555. We thank Nancy Elias-Rosa for initial analysis of the HST and CFHT images, and Mark Sullivan for information about the upper limit to the SN 2012aw/PTF12bvh detection by PTF. Some of the data presented herein were obtained at the W. M. Keck Observatory, which is operated as a scientific partnership among the California Institute of Technology, the University of California, and NASA; the observatory was made possible by the generous financial support of the W. M. Keck Foundation. A.G. is supported by grants from the ISF, BSF, and GIF foundations, a Weizmann Minerva grant, and the Lord Sieff of Brimpton Fund. The research of A.V.F. and his group at UC Berkeley is funded by Gary and Cynthia Bengier, the Richard and Rhoda Goldman Fund, NSF grant AST-0908886, the TABASGO Foundation, and NASA/HST grant AR-12623 from STScI. This work was supported in part by the NSF grant PHY-1066293 and the hospitality of the Aspen Center for Physics. We dedicate this paper to the memory of our dear friend and colleague, Weidong Li, with whom two of us (A.V.F. and S.D.V.) spent many fun years identifying the progenitor stars of $\mathrm{SNe}$; his tragic passing deeply saddened those who knew him.

\section{REFERENCES}

Arnett, W. D., Bahcall, J. N., Kirshner, R. P., \& Woosley, S. E. 1989, ARA\&A, 27, 629

Asplund, M., Grevesse, N., \& Sauval, A. J. 2005, in ASP Conf. Ser. 336, Cosmic Abundances as Records of Stellar Evolution and Nucleosynthesis, ed. T. G. Barnes, III \& F. N. Bash (San Francisco, CA: ASP), 25

Barbon, R., Ciatti, F., \& Rosino, L. 1979, A\&A, 72, 287

Baron, E., Branch, D., Hauschildt, P. H., et al. 2000, ApJ, 545, 444

Bessell, M. S. 1990, PASP, 102, 1181

Bessell, M. S., Castelli, F., \& Plez, B. 1998, A\&A, 333, 231

Bester, M., Danchi, W. C., Hale, D., et al. 1996, ApJ, 463, 336

Cardelli, J. A., Clayton, G. C., \& Mathis, J. S. 1989, ApJ, 345, 245

Chiavassa, A., Haubois, X., Young, J. S., et al. 2010, A\&A, 515, A12

Clocchiatti, A., Benetti, S., Wheeler, J. C., et al. 1996, AJ, 111, 1286

Crockett, R. M., Smartt, S. J., Pastorello, A., et al. 2011, MNRAS, 410, 2767

Danchi, W. C., Bester, M., Degiacomi, C. G., Greenhill, L. J., \& Townes, C. H. 1994, AJ, 107, 1469

Dolphin, A. E. 2000a, PASP, 112, 1383

Dolphin, A. E. 2000b, PASP, 112, 1397

Dwek, E., Arendt, R. G., Bouchet, P., et al. 2008, ApJ, 676, 1029

Ekström, S., Georgy, C., Eggenberger, P., et al. 2012, A\&A, 537, A146

Eldridge, J. J., Izzard, R. G., \& Tout, C. A. 2008, MNRAS, 384, 1109

Elias-Rosa, N., Van Dyk, S. D., Cuillandre, J.-C., et al. 2012, ATel, 3991

Elias-Rosa, N., Van Dyk, S. D., Li, W., et al. 2009, ApJ, 706, 1174 (erratum 711,1343 [2010])

Elmhamdi, A., Danziger, I. J., Chugai, N., et al. 2003, MNRAS, 338, 939

Fraser, M., Ergon, M., Eldridge, J. J., et al. 2011, MNRAS, 417, 1417

Fraser, M., Maund, J. R., Smartt, S. J., et al. 2012a, ATel, 3994

Fraser, M., Maund, J. R., Smartt, S. J., et al. 2012b, ApJ, submitted (arXiv:1204.1523)

Freedman, W. L., Madore, B. F., Gibson, B. K., et al. 2001, ApJ, 553, 47 Fruchter, A. S., \& Hook, R. N. 2002, PASP, 114, 144

Gustafsson, B., Edvardsson, B., Eriksson, K., et al. 2008, A\&A, 486, 951 Hamuy, M., Pinto, P. A., Maza, J., et al. 2001, ApJ, 558, 615

Hendry, M. A., Smartt, S. J., Crockett, R. M., et al. 2006, MNRAS, 369, 1303 Immler, S, \& Brown, P. J. 2012, ATel, 3995

Inserra, C., Turatto, M., Pastorello, A., et al. 2011, MNRAS, 417, 261

Josselin, E., \& Plez, B. 2007, A\&A, 469, 671

Kiss, L. L., Szabó, G. M., \& Bedding, T. R. 2006, MNRAS, 372, 1721

Knapen, J. H., de Jong, R. S., Stedman, S., \& Bramich, D. M. 2003, MNRAS, 344,527

Koekemoer, A. M., Fruchter, A. S., Hook, R. N., \& Hack, W. 2003, in The 2002 HST Calibration Workshop: Hubble after the Installation of the ACS and the NICMOS Cooling System, ed. S. Arribas, A. Koekemoer, \& B. Whitmore (Baltimore, MD: Space Telescope Science Institute), 337

Law, N. M., Kulkarni, S. R., Dekany, R. G., et al. 2009, PASP, 121, 1395

Leggett, S. K., Currie, M. J., Varricatt, W. P., et al. 2006, MNRAS, 373, 781

Leonard, D. C., Filippenko, A.V., Gates, E. L., et al. 2002, PASP, 114, 35

Leonard, D. C., Kanbur, S. M., Ngeow, C. C., \& Tanvir, N. R. 2003, ApJ, 594, 247

Leonard, D. C., Pignata, G., Dessart, L., et al. 2012, ATel, 4033

Levesque, E. M., Massey, P., Olsen, K. A. G., \& Plez, B. 2007, ApJ, 667, 202

Levesque, E. M., Massey, P., Olsen, K. A. G., et al. 2005, ApJ, 628, 973

Li, W., Van Dyk, S. D., Filippenko, A. V., \& Cuillandre, J.-C. 2005, PASP, 117,121

Li, W., Van Dyk, S. D., Filippenko, A. V., et al. 2006, ApJ, 641, 1060

Maguire, K., Di Carlo, E., Smartt, S. J., et al. 2010, MNRAS, 404, 981

Massey, P., Levesque, E. M., Olsen, K. A. G., Plez, B., \& Skiff, B. A. 2007, ApJ, 660,301 
Massey, P., Plez, B., Levesque, E. M., et al. 2005, ApJ, 634, 1286

Mattila, S., Smartt, S. J., Eldridge, J. J., et al. 2008, ApJ, 688, L91

Maund, J. R., \& Smartt, S. J. 2005, MNRAS, 360, 288

Maund, J. R., Smartt, S. J., \& Danziger, I. J. 2005, MNRAS, 364, L33

Monet, D. G., Levine, S. E., Canzian, B., et al. 2003, AJ, 125, 984

Moustakas, J., Kennicutt, R. C., Jr., Tremonti, C. A., et al. 2010, ApJS, 190, 233

Munari, U. 2012, CBET, 3054

Noriega-Crespo, A., van Buren, D., Cao, Y., \& Dgani, R. 1997, AJ, 114, 837

Pastorello, A., Zampieri, L., Turatto, M., et al. 2004, MNRAS, 347, 74

Persson, S. E., Murphy, D. C., Krzeminski, W., Roth, M., \& Rieke, M. J. 1998, AJ, 116, 2475

Pilyugin, L. S., \& Thuan, T. X. 2005, ApJ, 631, 231

Poznanski, D., Butler, N., Filippenko, A. V., et al. 2009, ApJ, 694, 1067

Poznanski, D., Ganeshalingam, M., Silverman, J. M., \& Filippenko, A. V. 2011, MNRAS, 415, L81

Poznanski, D., Nugent, P. E., Ofek, E. O., Gal-Yam, A., \& Kasliwal, M. M. 2012a, ATel, 3996, 1

Poznanski, D., Prochaska, J. X., \& Bloom, J. S. 2012b, MNRAS, submitted (arXiv:1206.6107)

Rahmer, G., Smith, R., Velur, V., et al. 2008, Proc. SPIE, 7014, 70144Y
Rau, A., Kulkarni, S. R., Law, N. M., et al. 2009, PASP, 121, 1334

Schlegel, D. J., Finkbeiner, D. P., \& Davis, M. 1998, ApJ, 500, 525

Simons, D. A., \& Tokunaga, A. 2002, PASP, 114, 169

Siviero, A., et al. 2012, CBET, 3054

Smartt, S. J., Eldridge, J. J., Crockett, R. M., \& Maund, J. R. 2009, MNRAS, 395, 1409

Smartt, S. J., Gilmore, G. F., Tout, C. A., \& Hodgkin, S. T. 2002, ApJ, 565,1089

Smartt, S. J., Maund, J. R., Hendry, M. A., et al. 2004, Science, 303, 499

Smith, N., Li, W., Filippenko, A. V., \& Chornock, R. 2011, MNRAS, 412, 1522

Stetson, P. B. 1987, PASP, 99, 191

Stockdale, C. J., Ryder, S. D., Van Dyk, S. D., et al. 2012, ATel, 4012

Tokunaga, A. T., Simons, D. A., \& Vacca, W. D. 2002, PASP, 114, 180

Van Dyk, S. D., Davidge, T. J., Elias-Rosa, N., et al. 2012, AJ, 143, 19

Van Dyk, S. D., Li, W., \& Filippenko, A. V. 2003, PASP, 115, 1289

Vogt, S. S., Allen, S. L., Bigelow, B. C., et al. 1994, Proc. SPIE, 2198, 362

Wesson, R., Barlow, M. J., Ercolano, B., et al. 2010, MNRAS, 403, 474

Woosley, S. E., \& Weaver, T. A. 1986, ARA\&A, 24, 205

Yadav, N., Chakraborti, S., \& Ray, A. 2012, ATel, 4010

Yoon, S.-C., \& Cantiello, M. 2010, ApJ, 717, L62

Zampieri, L., Pastorello, A., Turatto, M., et al. 2003, MNRAS, 338, 711 